\title{
Toxoplasma Pneumonia
}

National Cancer Institute

\section{Source}

National Cancer Institute. Toxoplasma Pneumonia. NCI Thesaurus. Code C35396.

Pneumonia caused by the parasite Toxoplasma gondii. It usually presents as an

opportunistic infection in immunocompromised patients, especially patients with AIDS. It is rare in immunocompetent individuals. Signs and symptoms include cough, fever, and dyspnea. 